\title{
Extended Daily Schedule of Temozolomide in Recurrent Glioblastoma: Single-Institution Report on a Series of 43 Patients
}

\author{
Alessandro D’Elia ${ }^{1 *}$, Vincenza Maiola ${ }^{1}$, Mirena Manucci ${ }^{1}$, Angelo Pichierri ${ }^{1}$, Alessandro Frati ${ }^{1,2}$, \\ Felice Giangaspero $^{1,2}$, Maurizio Salvati ${ }^{1,2}$ \\ ${ }^{1}$ Neurosurgery, Department of Neurological Sciences, Policlinico Umberto I, University of Rome “Sapienza”, Viale del Policlinico, \\ Rome, Italy; ${ }^{2}$ Department of Neurosurgery-INM Neuromed IRCCS, Via Atinense, Pozzilli, Italy. \\ Email: deliaale@gmail.com
}

Received November $18^{\text {th }}, 2013$; revised December $18^{\text {th }}, 2013$; accepted December $26^{\text {th }}, 2013$

Copyright (C) 2014 Alessandro D’Elia et al. This is an open access article distributed under the Creative Commons Attribution License, which permits unrestricted use, distribution, and reproduction in any medium, provided the original work is properly cited. In accordance of the Creative Commons Attribution License all Copyrights (C) 2014 are reserved for SCIRP and the owner of the intellectual property Alessandro D’Elia et al. All Copyright (C 2014 are guarded by law and by SCIRP as a guardian.

\begin{abstract}
Background: Despite advances in surgical and first-line adjuvant treatment, glioblastoma multiforme (GBM) always recurs as disease natural history. Currently, there is no consensus as to the optimal second-line treatment of recurrent GBM. Patients and Methods: This is a retrospective study of a series of adult patients consecutively treated at a single institution for supratentorial cerebral GBM at first relapse. All patients had previously received the standard concomitant radiochemotherapy protocol as first-line therapy. At recurrence/progression, all patients were treated with a metronomic temozolomide (TMZ) schedule at a daily dosage of $50 \mathrm{mg} / \mathrm{m}^{2} \mathrm{of}$ body surface. Radiologic, clinical, and laboratory data were collected for all patients, with a minimum follow-up of 18 months. Results: From January 2010 to June 2011, 43 patients were treated at our facility. A mean of 10 metronomic TMZ cycles (range, 3 - 21) was administered. Radiologically, we observed 2 complete responses (4.6\%), 16 partial responses $(37.2 \%)$, 18 stable disease $(41.9 \%)$ and 7 progressive disease (16.3\%). Steroids administration was safely tapered in 23 patients (53.5\%). Karnofsky-Performance-Status (KPS) results improved in 20 patients (46.5\%), stabilized in 20 (46.5\%), and worsened in 3 patients $(7.0 \%)$, with a mean KPS score increased from 65.1 at baseline to 75.3 at follow-up. Six-month progression-free survival was 53.5. One year after recurrence/progression diagnosis, 22 patients were still alive, with a 1-year overall survival rate of $51.6 \%$. Conclusions: The proposed TMZ schedule seems a safe and effective option for patients with recurrent GBM, with high radiologic response rates and good clinical impact. Strict clinical observation of patients may enable obtaining better results than those already present in the literature and further investigation appears auspicable.
\end{abstract}

\section{KEYWORDS}

Glioblastoma; Recurrence; Progression; Temozolomide; Metronomic Chemotherapy

\section{Introduction}

Glioblastoma multiforme (GBM, grade IV according to the World Health Organization classification system) [1] is the most common and most malignant primitive cerebral tumor in the adult. Despite recent advances in surgical management and first-line adjuvant treatment, recurrence is always expected, being part of the natural history

"Corresponding author. of the disease. The recurrence process of a GBM develops quite invariably within 2 years from the diagnosis, and it accounts for patient clinical deterioration and death, which often occur in a few weeks or months. Only recently, it achieved a general consensus with regard to adjuvant treatment following surgical intervention (firstline therapy). In fact temozolomide (TMZ), given concomitantly and subsequently to conformational fractionated radiotherapy, has become a standard of care for 
GBM patients [2-4]. By contrast, for patients with recurrent or progressive disease, there is still no consensus as to the optimal second-line treatment of GBM recurrent patients, and many options are generally considered for each patient. A second surgery, or a second irradiation with or without gamma-knife radiosurgery, is not always feasible, and a second-line chemotherapy strategy is often the treatment of choice. Nowadays, many alternative drugs are used in clinical practice-as in the experimental setting-such as lomustine and procarbazine, fotemustine, irinotecan and bevacizumab, tamoxifen, celecoxib, erlotinib, cediranib, and cilengitide, alone or in combination; even TMZ is considered as second-line chemotherapy with different schedules [3,4]. Unfortunately, these therapies show a limited response rate, and, even when effective, the duration of response is short and does not always result in improved overall survival (OS) or quality of life.

We present here our experience with a metronomic schedule of daily administration of TMZ, employed at a dosage of $50 \mathrm{mg} / \mathrm{m}^{2}$ of body surface, in patients with recurrent supratentorial adults GBM who were previously treated with standard adjuvant TMZ until disease progression.

\section{Patients and Methods}

This is a retrospective study of a series of adult patients consecutively treated at a single institution for histologically proven supratentorial cerebral GBM at first relapse. All patients received conformational fractionated (54 - 66 Gy delivered in 30 fractions, 1.8 - 2.2 Gy per fraction) or hypofractionated (three cases) adjuvant radiotherapy and concomitant TMZ (75 mg/m $\mathrm{m}^{2}$ of body surface), and subsequent TMZ (200 mg/m $\mathrm{m}^{2}$ of body surface, days 1 to 5 of every 28-day cycle) chemotherapy, according to the standard schedule. Recurrent or progressive disease was diagnosed by conventional contrast-enhanced magnetic resonance imaging (MRI) scan performed about 40 days after the completion of concomitant radiochemotherapy or every 3 months during follow-up. To exclude pseudoprogression, adjuvant second-line treatment was never started before 3 months after the completion of concomitant radiochemotherapy and steroid therapy; a 1-month successive MRI study was performed with metabolic analysis performed by means of spectroscopy and/or regional cerebral blood volume (rCBV) perfusion analysis before adjuvant second-line metronomic TMZ administration in the majority of cases. Inclusion criteria comprised the completion of a radiochemotherapy regimen, patient compliance with an oral therapy administration, and adequate laboratory values, in particular a platelet count of $\geq 10,000 / \mathrm{dL}$, neutrophilic count of $\geq 2000 / \mathrm{dL}$, lymphocytes $\geq 800 / \mathrm{dL}$, red blood cells $\geq 3.5 \times 10^{6} / \mathrm{dL}$ with hemoglobin $\geq 9 \mathrm{~g} / \mathrm{dL}$, serum creatine $\leq 1.5$ times the upper limit of laboratory normal (ULLN), aspartate transaminase or alanine transaminase $\leq 2.5$ times ULLN, and alkaline phosphatase $\leq 3.0$ times ULLN. After disease confirmation, TMZ was administered continuously at $50 \mathrm{mg} /$ $\mathrm{m}^{2}$ of body surface daily, rounded to the nearest $5 \mathrm{mg}$, and patients were seen monthly in clinical follow-up. Routine laboratory examinations were performed weekly, and therapy discontinuation days and hematologic toxicities were recorded. Routine MRI scans were performed every 3 months, and responses were recorded according to Macdonald criteria (see discussion) as complete response (CR), partial response (PR), stable disease (SD) or progressive disease (PD). Clinical response data on KPS and changes in the need for steroids were also recorded. Duration of responses was registered until disease progression, and the regimen was administered for a minimum of 3 cycles until tumor progression or stabilization on metabolic MRI study.

Patients who were lost at follow-up were included in the statistical analysis for progression-free survival (PFS) and overall survival (OS): they were considered as progression and death at the date of last follow-up.

Statistical analysis: The Pearson chi-squared tests were used to evaluate associations in frequency tables. Survival curves according to Kaplan-Meyer method were obtained and the log-rank test was used to evaluate differences between two survival curves.

The extent of tumor removal at GBM diagnosis was classified as follows: Grossly total (GTR): no evidence of residual disease both with intraoperative microscopic examination and with early brain postcontrast MRI; subtotal: $>90 \%$ and $<100 \%$; partial: $<90 \%$; biopsy: $<50 \%$.

All patients gave their written informed consent to the treatment.

MGMT promoter methylation status was evaluated with methylation-specific PCR on DNA extracted from formalin-fixed, paraffin-embedded samples, obtained from the surgical specimen of all 43 patients.

\section{Results}

In the period from January 2010 to June 2011, 43 patients were treated at the Neurosciences Department Neurosurgery of Sapienza University of Rome. Mean age was 57.2 years (range, 39 - 82 years), with a median age of 57.5 years; 25 patients were males and 18 were females (male: female ratio $=1.4: 1$ ). Median KPS at baseline was 65 (range, 40 - 100, 95\% C.I. $65.1 \pm 5.3$ ). The most frequently involved primary site was the frontal lobe (25 cases, 58.1\%), followed by a temporal site in 19 patients (44.2\%); in 11 patients (25.6\%) the tumor presented as multifocal. Surgical removal at first surgery was GTR in 18 cases (41.9\%), subtotal in 12 cases 
(27.9\%), partial in 5 cases (11.6\%) and only biopsy in 8 cases (18.6\%). In all cases, concomitant adjuvant radiation and chemotherapy were completed according to the standard Stupp protocol (60 Gy delivered in 30 fractions in a 6-week period, and concomitant administration of TMZ 1 hour before radiotherapy, at the dosage of $75 \mathrm{mg} / \mathrm{m}^{2}$ of body surface), except for 3 patients, for whom a hypofractionated regimen was administered (30 Gy in 10 fractions over a 2-week period for 2 patients; 40 Gy in 15 fractions over a 3-week period for the other patient, each time accompanied by the same TMZ regimen-see Table 1). After a median time of 34 days (range, 21 - 45 days, 95\% C.I. $34.2 \pm 4.4$ ) after the completion of radiochemotherapy, a standard chemotherapy regimen was started with TMZ: $200 \mathrm{mg} / \mathrm{m}^{2}$ of body surface administered on days 1 to 5 of every 28-day cycle, until disease progression, with a median number of 5.9 cycles (range 1 - 28 cycles, 95\% C.I. $5.9 \pm 2.1$ ). The median time to progression was 10.9 months (range, 0 - 45 months, 95\% C.I $10.9 \pm 6.4$ ).

After disease recurrence/progression, at least 18 months of follow-up was achieved for all but 1 patient, a 41year-old man who refused further therapy and examination 12 months after disease progression and treatment. As second-line therapy, a mean of 9 TMZ 28-day cycles (range, 3 - 21 cycles, 95\% C.I. $9.2 \pm 1.4$ ) was administered continuously at the daily dosage of $50 \mathrm{mg} / \mathrm{m}^{2}$ of body surface. The programmed MRI examinations were performed in all cases, and according to Macdonald criteria, we observed 2 CR (4.6\%), 16 PR (37.2\%), 18 SD (41.9\%), and 7 PD (16.3\%). Steroids administration was tapered without adjunctive symptoms in 23 patients (53.5\%), but clinical response did not allow steroids tapering in 8 patients (18.6\%); in the remaining cases patients had no need for steroids. KPS analysis revealed an improved score for 20 patients (46.5\%), stabilization for 20 patients (46.5\%) and worsening in 3 patients (7.0\%), with a mean KPS score that increased from 65.1 at baseline to 75.3 at follow-up (95\% C.I. $75.3 \pm 5.5)$. The 6-month PFS (PFS6) for all patients was 53.5\%. The 52week progression-free Kaplan-Meier curve is shown in Figure 1; at 1 year, 6 patients (14.0\%) were still progression-free (Table 2, Figure 1). One year after recurrence/progression diagnosis, 22 patients were still alive (1-year OS, 51.6\%). The 18-month OS Kaplan-Meier curve is shown in Figure 2; 10 patients (23.3\%) were still alive after 18 months (Table 2).

About MGMT gene promoter status, for 26 patients it resulted methylated whereas for 17 patients it was not methylated. Subgroup analysis showed a median time to progression of 12.0 months (95\% C.I. $12.0 \pm 5.2$ ) in the methylated group and 9.6 months (95\% C.I. $9.6 \pm 4.8$ ) for the not-methylated group, and the difference was sta-

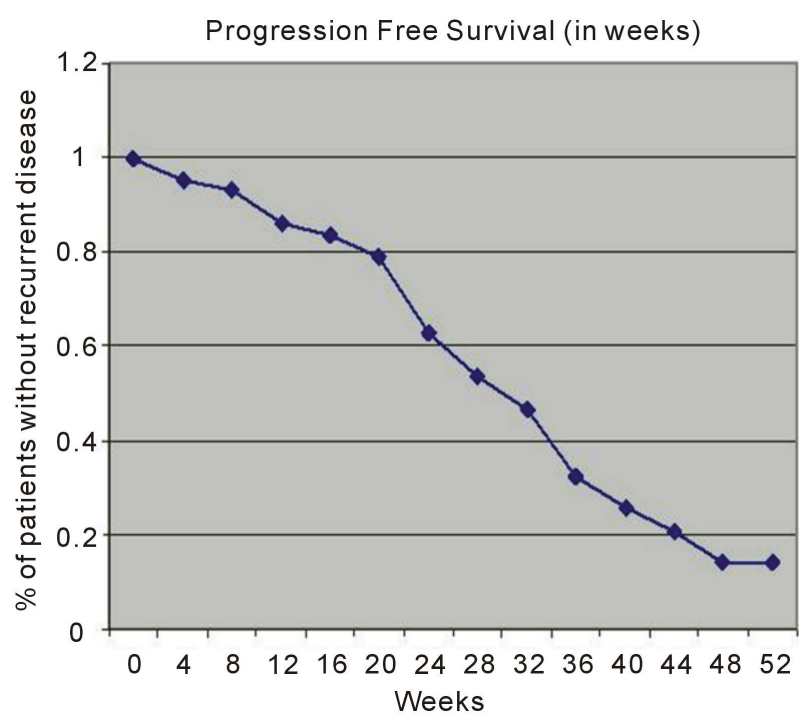

Figure 1. Kaplan-Meier plot of progression free survival (PFS) after study enter (in weeks). At 26 weeks (that means at 6 months) the progression-free survival is $53.5 \%$, as shown in the text.

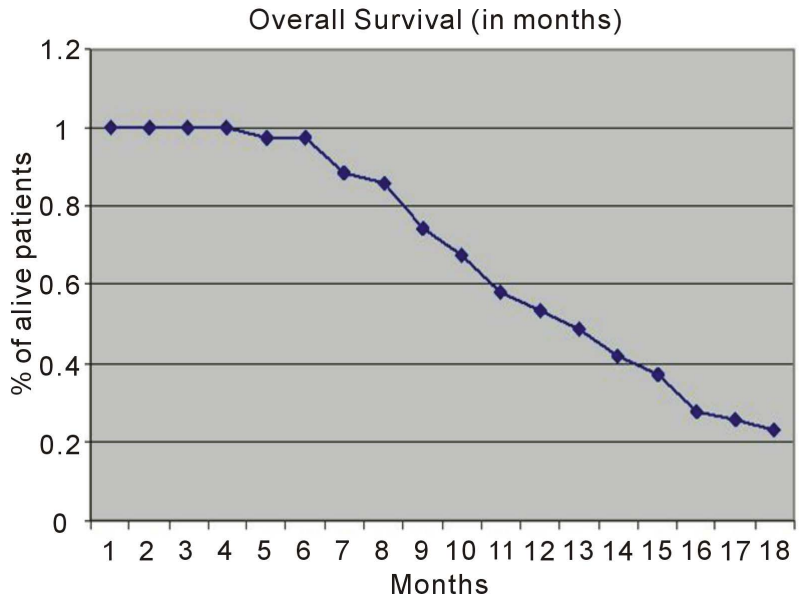

Figure 2. Kaplan-Meier plot of overall survival (OS) after study enter (in months). As it is shown, all but one patients were still alive at six months from enrolment, and at 1 year 22 patients representing $51.6 \%$ are still alive.

tistically significative $(\mathrm{p}=0.03)$ (Figure 3$)$. About the other data under investigation, no correlation was found between MGMT status and response to II line therapy, PFS and OS (Figure 4) after study entry.

\section{Discussion}

Although first-line chemotherapy for GBM patients is now well established, recurrence is always expected, and related treatment still remains a challenge. For the majority of GBM patients, there is no unequivocal treatment proposal at the time of the recurrence, leading to consid- 
Table 1. Clinical characteristics of all patients. GTR = Grossly Total Removal; subtot = subtotal removal; hypo = hypofractionated regimen (lower number of fractions with higher dosage for each fraction); MET or NON MET = methylated or not methylated status of MGMT gene promoter.

\begin{tabular}{|c|c|c|c|c|c|c|c|c|}
\hline Age/gender & Site & Hystology & asportation & $R T+T M Z$ & $n^{\circ} 5 / 28$ cycles & MGMT status & baseline KPS & KPS at examination \\
\hline $74 \mathrm{M}$ & T sn & GBM & subtot & Yes & 4 & MET & 70 & 100 \\
\hline $61 \mathrm{~F}$ & PT dx & GBM & subtot & Yes & 1 & NON MET & 70 & 70 \\
\hline $53 \mathrm{M}$ & $\mathrm{Odx}$ & GBM & subtot & Yes & 2 & MET & 60 & 60 \\
\hline $60 \mathrm{M}$ & F sn & GBM & biopsy & Нуро $10 \times 3$ & 3 & MET & 50 & 100 \\
\hline $56 \mathrm{M}$ & $\mathrm{F} \mathrm{dx}$ & GBM & biopsy & Нуро $15 \times 2.66$ & 1 & NON MET & 50 & 70 \\
\hline $67 \mathrm{~F}$ & T sn & GBM & GTR & Yes & 5 & MET & 50 & 50 \\
\hline $45 \mathrm{M}$ & $P d x$ & GBM & GTR & Yes & 9 & MET & 100 & 100 \\
\hline $47 \mathrm{M}$ & F sn & GBM & GTR & Yes & 21 & MET & 50 & 40 \\
\hline $59 \mathrm{M}$ & F sn & GBM & GTR & Yes & 12 & NON MET & 80 & 80 \\
\hline $58 \mathrm{~F}$ & T sn & GBM & subtot & Yes & 3 & MET & 50 & 70 \\
\hline $39 \mathrm{~F}$ & F sn & GBM & GTR & Yes & 9 & MET & 100 & 100 \\
\hline $41 \mathrm{M}$ & F sn & GBM & partial & Yes & 1 & NON MET & 50 & 100 \\
\hline $65 \mathrm{~F}$ & F sn & GBM & subtot & Yes & 1 & NON MET & 50 & 50 \\
\hline $66 \mathrm{~F}$ & $\mathrm{~T}$ sn & GBM & GTR & Yes & 1 & MET & 60 & 60 \\
\hline $41 \mathrm{M}$ & FT sn & GBM & biopsy & Yes & 9 & NON MET & 50 & 70 \\
\hline $56 \mathrm{~F}$ & F sn & GBM & biopsy & No & 1 & NON MET & 40 & 60 \\
\hline $39 \mathrm{M}$ & F sn & GBM & partial & Yes & 18 & MET & 90 & 100 \\
\hline $55 \mathrm{M}$ & FTP dx & GBM & GTR & Yes & 1 & MET & 50 & 90 \\
\hline $61 \mathrm{~F}$ & T sn & GBM & GTR & Yes & 28 & MET & 60 & 40 \\
\hline $57 \mathrm{~F}$ & $\mathrm{Fdx}$ & GBM & GTR & Yes & 2 & NON MET & 80 & 80 \\
\hline $44 \mathrm{~F}$ & F sn & GBM & subtot & Yes & 1 & MET & 60 & 70 \\
\hline $43 \mathrm{~F}$ & FT ins dx & GBM & subtot & Yes & 2 & NON MET & 60 & 60 \\
\hline $44 \mathrm{M}$ & FT dx & GBM & subtot & Yes & 1 & MET & 60 & 100 \\
\hline $54 \mathrm{M}$ & $\mathrm{T}$ sn & GBM & GTR & Yes & 3 & MET & 60 & 80 \\
\hline $40 \mathrm{~F}$ & $\mathrm{~F} \mathrm{dx}$ & GBM & partial & Yes & 3 & NON MET & 50 & 70 \\
\hline $70 \mathrm{~F}$ & FP sn & GBM & GTR & Yes & 18 & NON MET & 50 & 50 \\
\hline $60 \mathrm{M}$ & $\mathrm{T}$ sn $\mathrm{P} d \mathrm{x}$ & GBM & partial & Yes & 3 & NON MET & 50 & 100 \\
\hline $47 \mathrm{~F}$ & splenio & GBM & biopsy & Yes & 2 & MET & 70 & 70 \\
\hline $58 \mathrm{M}$ & FT sn & GBM & subtot & Yes & 18 & MET & 50 & 70 \\
\hline $71 \mathrm{M}$ & $\mathrm{T} \mathrm{dx}$ & GBM & GTR & Yes & 3 & MET & 100 & 100 \\
\hline $65 \mathrm{M}$ & $\mathrm{F} \mathrm{dx}$ & GBM & GTR & Yes & 12 & MET & 90 & 90 \\
\hline $56 \mathrm{~F}$ & $\mathrm{Odx}$ & GBM & GTR & Yes & 2 & NON MET & 100 & 100 \\
\hline $65 \mathrm{M}$ & F sn & GBM & GTR & Yes & 24 & MET & 60 & 70 \\
\hline $63 \mathrm{~F}$ & F sn & GBM & GTR & Yes & 2 & NON MET & 60 & 70 \\
\hline $82 \mathrm{M}$ & $\mathrm{F} \mathrm{dx}$ & GBM & biopsy & Нуро $10 \times 3$ & 3 & MET & 60 & 60 \\
\hline $75 \mathrm{M}$ & F bilat & GBM & biopsy & Yes & 1 & NON MET & 50 & 50 \\
\hline $48 \mathrm{M}$ & P sn & GBM & subtot & Yes & 2 & MET & 70 & 70 \\
\hline $60 \mathrm{~F}$ & $\mathrm{Tdx}$ & GBM & GTR & Yes & 6 & MET & 70 & 90 \\
\hline $55 \mathrm{~F}$ & $\mathrm{~T}$ sn & GBM & subtot & Yes & 1 & MET & 60 & 80 \\
\hline $70 \mathrm{M}$ & $F d x$ & GBM & GTR & Yes & 2 & NON MET & 80 & 80 \\
\hline $66 \mathrm{M}$ & T sn & GBM & biopsy & Yes & 1 & MET & 40 & 60 \\
\hline $71 \mathrm{M}$ & $\mathrm{Tdx}$ & GBM & partial & Yes & 3 & NON MET & 100 & 100 \\
\hline $52 \mathrm{M}$ & $\mathrm{Tdx}$ & GBM & subtot & Yes & 9 & MET & 90 & 60 \\
\hline
\end{tabular}



on a Series of 43 Patients

Table 2. Treatment related data. $\mathbf{C R}=$ Complete Response; $\mathbf{P R = P r o g r e s s i v e ~}$ Disease; $\mathbf{S D}=$ Stable Disease; $\mathbf{P D}=\mathbf{P r o g r e s s i v e}$ Disease (see the text for details); PFS = Progression-Free Survival; OS = Overall Survival.

\begin{tabular}{|c|c|c|c|c|c|c|c|c|c|}
\hline $\begin{array}{c}n^{\circ} 28 / 28 \\
\text { cycles }\end{array}$ & $\begin{array}{c}\text { KPS } \\
\text { change }\end{array}$ & $\begin{array}{c}\text { radiologic } \\
\text { response }\end{array}$ & $\begin{array}{c}\text { tapering } \\
\text { of steroids }\end{array}$ & PFS 6 & $\begin{array}{c}P F S \\
\text { (weeks) }\end{array}$ & 1 year $O S$ & OS (months) & Hemathology & Disconti-nuation \\
\hline 14 & improved & PR & YES & YES & 48 & YES & 16 & & \\
\hline 13 & stable & SD & YES & NO & 28 & YES & 18 & & \\
\hline 3 & stable & PD & NO & NO & 0 & NO & 8 & & \\
\hline 18 & improved & CR & YES & YES & 64 & YES & $>18$ & grade III leukopenia & 7 days \\
\hline 12 & improved & PR & YES & YES & 38 & NO & 9 & & \\
\hline 18 & stable & SD & & YES & 60 & YES & $>18$ & & \\
\hline 18 & stable & PR & & YES & 52 & YES & $>18$ & & \\
\hline 6 & worsened & $\mathrm{PD}$ & NO & NO & 8 & NO & 6 & & \\
\hline 8 & stable & SD & & NO & 20 & YES & 17 & grade III neutropenia & 10 days \\
\hline 11 & improved & PR & YES & YES & 40 & NO & 11 & & \\
\hline 21 & stable & SD & & NO & 25 & YES & 14 & & \\
\hline 6 & improved & PR & YES & YES & 32 & YES & 12 & \multicolumn{2}{|c|}{ grade III anemia } \\
\hline 5 & stable & $\mathrm{PD}$ & NO & NO & 0 & NO & 6 & & \\
\hline 7 & stable & SD & & YES & 30 & YES & 16 & & \\
\hline 18 & improved & PR & YES & YES & 64 & YES & $>18$ & & \\
\hline 8 & improved & PR & YES & NO & 24 & NO & 8 & grade III leukopenia & 7 days \\
\hline 8 & improved & PR & YES & YES & 35 & NO & 11 & & \\
\hline 5 & improved & PR & YES & NO & 18 & NO & 6 & & \\
\hline 3 & worsened & PD & NO & NO & 6 & NO & 4 & & \\
\hline 18 & stable & SD & & YES & 36 & YES & $>18$ & & \\
\hline 6 & improved & SD & & NO & 23 & NO & 10 & grade III linphopenia & 7 days \\
\hline 6 & stable & SD & YES & NO & 22 & NO & 8 & grade III ar & nemia \\
\hline 12 & improved & PR & YES & YES & 48 & YES & $>18$ & & \\
\hline 8 & improved & SD & YES & YES & 22 & NO & 10 & & \\
\hline 8 & improved & PR & NO & NO & 26 & YES & 12 & & \\
\hline 8 & stable & SD & & YES & 76 & YES & $>18$ & & \\
\hline 10 & improved & PR & YES & YES & 38 & YES & 13 & & \\
\hline 9 & stable & PR & NO & YES & 35 & NO & 10 & & \\
\hline 7 & improved & SD & YES & NO & 20 & NO & 7 & & \\
\hline 9 & stable & SD & YES & YES & 32 & YES & 13 & & \\
\hline 7 & stable & PR & YES & YES & 35 & YES & 13 & & \\
\hline 8 & stable & SD & & NO & 20 & YES & 14 & & \\
\hline 6 & improved & SD & YES & NO & 8 & YES & 16 & & \\
\hline 7 & improved & SD & & YES & 30 & YES & 16 & grade III thrombopenia & 15 days \\
\hline 4 & stable & SD & NO & NO & 20 & NO & 9 & & \\
\hline 5 & stable & PD & YES & NO & 25 & NO & 8 & & \\
\hline 11 & stable & PR & YES & YES & 40 & YES & $>18$ & & \\
\hline 12 & improved & PR & & YES & 48 & YES & $>18$ & & \\
\hline 12 & improved & CR & YES & YES & 55 & YES & $>18$ & & \\
\hline 4 & stable & $\mathrm{PD}$ & NO & NO & 9 & NO & 10 & & \\
\hline 4 & improved & SD & YES & NO & 16 & NO & 6 & & \\
\hline 9 & stable & SD & & YES & 35 & NO & 9 & & \\
\hline 5 & worsened & $\mathrm{PD}$ & YES & NO & 15 & NO & 8 & grade III linphopenia & 10 days \\
\hline
\end{tabular}

erable confusion and discouragement. Recurrence often represents the first step to neurologic deterioration and death.

The antitumor activity of TMZ has been shown to be schedule-dependent [4-7]. Given the unavoidable need for effective second-line treatment, we started considering new, alternative TMZ regimens, to take advantage of the excellent tolerability profile that this drug has shown 
MGMT gene promoter and time to disease progression

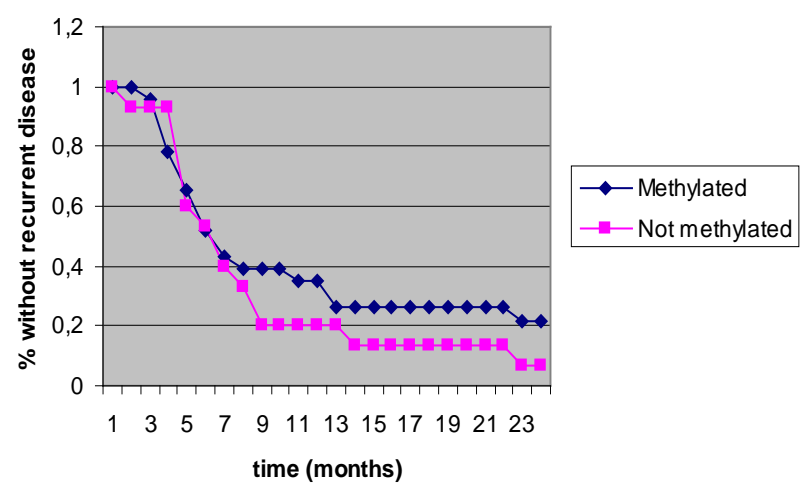

Figure 3. MGMT gene promoter status subgroup analysis of time to progression (in months) from the first diagnosis of GBM. As already known, MGMT correlates to time to progression of patients at first diagnosis $(p=0.03)$.

\section{OS and MGMT gene promoter methylation status}

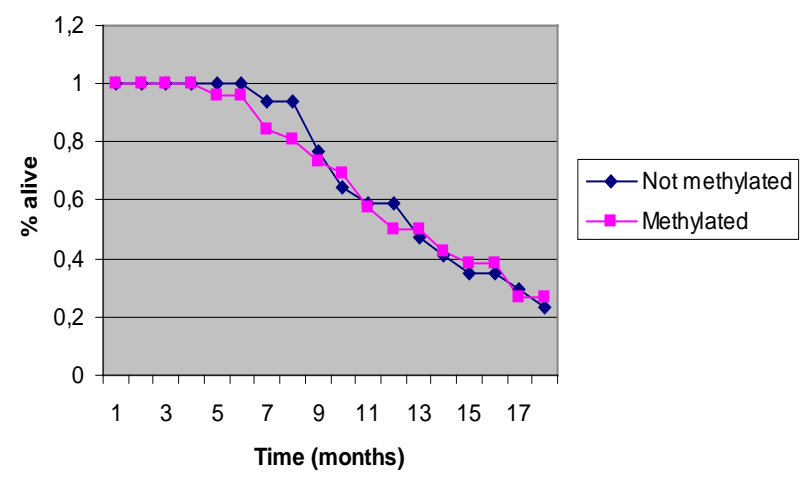

Figure 4. MGMT gene promoter status subgroup analysis of overall survival (OS) after study enter (in months). There is no significant difference in overall survival after disease relapse according to MGMT status at first diagnosis ( $p>$ $0.05)$, as to progression-free survival after recurrence.

in many studies [3,4,8-13] as in clinical daily use.

The role of TMZ in recurrent GBM patients [12,13] has been questioned: since 2005, when the European Organisation for Research and Treatment of Cancer multicenter study made the Stupp protocol a standard of care as first-line therapy [2-4], fewer and fewer patients have been TMZ-naïve at recurrence [14]. It is only in recent years that the TMZ rechallenge strategy has become an attractive option for recurrent GBM patients, since the publication of results from TMZ rechallenge studies [12] and the "rescue" approach by Perry and coworkers [13], together with results from new, alternative intensified TMZ schedules [3,4,9,10].

There are 2 main theoretical assumptions that make the metronomic TMZ schedule suitable for recurrent
GBM patients after standard TMZ treatment failure. The first is the possibility of TMZ administered with a protracted oral schedule to overcome the cellular DNA-repair systems mediated by O-6-methylguanine-DNA methyltransferase (MGMT). MGMT is a DNA-repairing enzyme that limits the mutagenicity and the cytotoxic effects of alkylating agents by removing the methyl group from $\mathrm{O}(6)$ of guanine, a site that is considered of key importance for nitrosoureas effectiveness [7,15]. MGMT is thus considered essential in inducing chemoresistance in tumor cells exposed to alkylating drugs [15-19]. During the last 10 years, several strategies have been developed with the intent to modulate MGMT activity $[7,15,18]$, and among these are metronomic TMZ schedules. When MGMT repairs O6-methylguanine lesions, it transfers the alkyl group from guanine to a cysteine in its active site, thereby repairing the DNA $[7,15,16]$. In this process, however, MGMT is irreversibly inactivated, such that new protein synthesis is required to restore function [15,23]. There are several laboratory studies in which MGMT activity was measured in peripheral blood mononuclear cells (PBMC models), along with the effect and kinetics of chemotherapeutic agents that are able to modulate its activity. Among these studies, Tolcher and colleagues [20] studied the effects of alternative TMZ regimens on MGMT activity. These authors found that continuous daily dosing of TMZ for 7 days at $75-175 \mathrm{mg} / \mathrm{m}^{2} /$ day reduced baseline MGMT activity by $72 \%$ on day 8 in a dose-dependent manner, and, more interesting, that during a rest period, MGMT activity recovered. The effect on MGMT activity depletion was more sustained with 21 days of continuous TMZ administration in 28 days, even at a lower daily dosage ( 85 - $125 \mathrm{mg} / \mathrm{m}^{2}$ ). These observations are of great significance, even though the PBMC models were subjected to some criticism; tumor tissue data of MGMT kinetic analyses are expected [4,15]. It remains unclear, however, whether the alternative TMZ schedules are more effective than the standard dosing regimen or whether they effectively deplete MGMT activity in tumor tissue [15].

The second assumption is the antiangiogenic properties of metronomic daily administration of TMZ. Endothelial proliferation is among the diagnostic hallmarks of GBM. Gliomas are highly angiogenic, and the higher grade tumors show evidence of greater angiogenic activity, with increased microvessel density and neovascularization [1,21]. Moreover, tumor vessel density appears to be an independent prognostic parameter for gliomas [22], and angiogenesis plays a critical role in the progression and clinical behavior of these tumors. In fact, the amount of surrounding brain tumor edema and the need for steroids is a direct consequence of the patho- 
logic vasculature. As tumor growth is critically dependent on the formation of new blood vessels, inhibition of this process has offered an attractive strategy to complement standard therapies $[3,23,24]$.

An alternative and immediately accessible strategy to inhibit angiogenesis is continuous chemotherapy administration, and also referred to as "metronomic chemotherapy” [3,4,23-25]. With such an approach, conventional cytotoxic chemotherapeutic agents are given nearly continuously at a lower dosages, disrupting rapidly proliferating tumor endothelium and preventing tumor growth [3,4,23-27]. Continuous administration of chemotherapy has shown an antiangiogenic effect that is partly explained by the effects on the endothelial progenitor cells $[25,28]$ and on thrombospondin-1, a mediator of antiangiogenic effects [29], and these effects appear common for metronomic TMZ regimens [4,13,24,27,30,31]. This feature of metronomic TMZ schedule enables us to interpret the high radiologic response rate we have obtained (83.7\%), which compares more closely with that obtained in glioblastoma clinical trials on vascular endothelial growth factor (VEGF) inhibitors [32-35]. In these studies, using Macdonald's criteria [36], radiologic response rates ranged from $28 \%$ to $53 \%$, which are similar to our results. Another peculiarity of the antiangiogenic treatment is the modality of tumor spread at disease progression under the antiangiogenic regimen [33,37]. The progression of disease appears on MRI scan with a diffuse spread, and this fact has been explained by the recruitment of normal cerebral vessels by the tumor. This modality of tumor spread after an initial clinical and radiologic response period was also observed in our series.

Even the possibility of tapering steroids represents a peculiarity of antiangiogenic treatments [3], and in the cited trials it was achieved in almost $50 \%$ of cases [32-35]. In our series, steroids administration was tapered without adjunctive symptoms in 23 patients (53.5\%), and this fact indirectly confirms the antiangiogenic effect of metronomic TMZ. The possibility for the patient to taper steroids is of great significance, because even in the presence of a stable or slowly progressive disease, it directly affects the quality of life. Tapering of steroids permits metabolism to normalize, maintaining body weight, reducing problems connected with steroids-related hyperglycemia and loss of bone density, and restoring immune system competence. So even though a neurologic examination seems unvarying over time, the lowering of steroid administration represents a sign of improved quality of life that is not readily quantifiable. This event occurred in 6 patients $(=14 \%)$ without other sign of response (see Table 2).

The efficacy of the protracted daily schedule of TMZ has been observed since the first studies addressing the safety and feasibility of the continuous low-dose regimen. For melanoma patients, disease stabilization on the continuous TMZ schedule was observed in patients who had progressed through the 5-day Phase II schedule [38]. In a later Phase I trial, Brock and colleagues [6] tried to determine the maximum tolerated daily dosage of TMZ administered for 6- to 7-week cycles, starting at 50 $\mathrm{mg} / \mathrm{m}^{2}$ of body surface. For 17 patients with gliomas who were enrolled in their study, 12 of whom had high-grade gliomas, they observed a $41 \%$ objective response, obtaining disease stabilization in 6 of 17 patients.

As recently pointed out [39], if radiologic response rate is assessed with Macdonald criteria, the radiologic results mainly represent an estimate of changes in contrast enhancement. That is an important clinical feature of the glioma, but there are other important pathologic features of the disease that correlate with patient outcome. These features include cellular density and effective tumor spread. The actual effectiveness of a second-line treatment for recurrent GBM will be better assessed with the 6-month PFS and OS indices [40,41].

To date, to our knowledge, there are only 4 clinical studies that used daily TMZ at $50 \mathrm{mg} / \mathrm{m}^{2}$ of body surface after conventional TMZ treatment failure. The first is a single-institution study by Perry and coworkers [13]. These authors divided GBM patients into 2 subgroups; only the second group compares with our series. There were 14 patients with recurrent GBM at first progression after concurrent chemoradiation with TMZ and adjuvant TMZ. These patients completed 6 cycles of adjuvant $\mathrm{TMZ}$ at $200 \mathrm{mg} / \mathrm{m}^{2}$ on days 1 to 5 every 28 days, and were followed up until progression. The results of metronomic TMZ therapy in this setting showed 2 PR (14\%) and 9 SD (64\%) according to Macdonald's criteria, with a 6-month PFS of $57 \%$ and some grade of lymphopenia in over half of the patients, although not associated with any clinical manifestation.

A second Phase II multicenter study followed [30], which was more similar to our study with respect to the number of treated cases. For 91 GBM patients at first recurrence treated with the same metronomic TMZ schedule, the overall results were a 6 -months PFS of $23.9 \%$ and a 1-year OS of $27.3 \%$. A further subanalysis was made according to the history of the disease, as follows: all patients received standard radiochemotherapy, and the first subgroup (33 patients) was that in which GBM progression happened while receiving standard adjuvant TMZ before completion of the six 28-day cycles. This group was termed "early," and showed a PFS6 of 27.3\% with a 1 -year OS of $14.8 \%$. For the second group, recurrence occurred while receiving extended adjuvant TMZ beyond the standard 6 cycles but before completion of adjuvant treatment. This subgroup (27 patients) was 
termed "extended"; it showed a PFS6 of 7.4\% with a 1-year OS of $28.6 \%$. The third was the "rechallenge" group (28 patients): progression of the disease happened after the completion of adjuvant treatment, with a treatment-free interval of more than 2 months. For the rechallenge subgroup, the PFS6 was $35.7 \%$, with a 1-year OS of $28.6 \%$. The objective response in the study was $3 \%, 0 \%$, and $11.1 \%$ respectively for the early, extended, and rechallenge groups.

In our study, there was no true adjuvant TMZ discontinuation, and TMZ was administered in the standard adjuvant fashion until disease progression (Table 1). Following the same temporal criteria, our data produce the following results: for the early subgroup (30 patients) PFS6 was $56.7 \%$ and 1-year OS was $50.0 \%$, for the extended subgroup (5 patients) PFS6 was 40\% and 1-year OS was $80 \%$, and for the rechallenge group without a TMZ discontinuation for more than 1 month (8 patients), PFS6 was $37.5 \%$ and 1 -year OS was $50.0 \%$. This subgroup analysis according to the temporal criteria used by Perry and colleagues is limited by the fact that the extended and rechallenge groups comprised only a few patients, and the great majority of patients were treated earlier in the disease history (30 patients, $70.0 \%$ of total).

These findings, however, deserve further examination. In fact, in the work by Perry et al., it is arguable that the favorable results related to the early subgroup may be in part attributable to pseudoprogression. Pseudoprogression is defined as the effect of the prior treatments on cerebral tissue causing transient increased permeability of vasculature and simulating a tumor recurrence, with radiologic evidence of contrast enhancement and digitated edema [39,42,43]. This phenomenon was well known in the past as "radionecrosis," but in recent years it has increased with the addition of TMZ to radiotherapy $[39,44,45]$. Therefore, Perry and coworkers tried to minimize the possible source of error due to pseudoprogression by excluding patients who experienced progression within 3 months of radiation therapy. In our series, to exclude pseudoprogression, adjuvant second-line treatment was never started before 3 months after the completion of concomitant radiochemotherapy. The 3-month interval is a minimum interval suggested by the international Response Criteria in Neuro-Oncology (RANO) working group $[39,46]$. Another empiric strategy we adopted to limit the pseudoprogression bias was the suspension of TMZ therapy and the early administration of dexamethasone, followed by a 1-month successive MRI study, to look for contrast enhancement changes. Whenever possible, MRI study was performed with metabolic analysis performed by means of spectroscopy and/or rCBV perfusion analysis before adjuvant second-line metronomic TMZ administration. Even though these advanced metabolic MRI tools are not completely validated, their integration with clinical data may be helpful in pseudoprogression diagnosis and differentiation $[47,48]$.

To date, the only tool that can unequivocally differentiate between tumor recurrence and pseudoprogression is the histopathologic examination when a second surgery is performed. In our series, this was done in only 7 cases (see Table 2), 5 of which were in the early subgroup. None of these examined cases showed a pseudoprogression. Another consideration is that in our series 8 patients where treated with only a biopsy at first surgery, and all but one of these patients were in the "early" group. Even considering these facts, pseudoprogression can not be unequivocally excluded for the majority of patients, and may in part account for the high response rate and best outcome of our patient series compared with the other cited studies.

In the third study, by Kong and colleagues [31], the metronomic daily administration of TMZ was subdivided in 2 cohorts: 10 patients received $40 \mathrm{mg} / \mathrm{m}^{2}$ of body surface and 28 patients received $50 \mathrm{mg} / \mathrm{m}^{2}$ of body surface. The overall PFS6 in all 38 patients was $32.5 \%$, with a median OS of 41 weeks. According to Macdonald criteria, PR was observed in 2 patients and SD in 21 patients, adding up to an overall radiologic response rate of $60.5 \%$. In our series we observed 2 CR (4.6\%), 16 PR (37.2\%), and 18 SD (41.9\%), for an overall radiologic response rate of $83.7 \%$. As pointed out previously, even this result may be in part due to the possibility of pseudoprogression. We believe it is noteworthy, however, that we observed CR in 2 patients, one of whom was diagnosed by a biopsy, where leaving all the tumor mass was left over the basal ganglia and internal capsule (Figure 5). To date, a CR has not been reported with this kind of second-line treatment.

Kong et al. [31] reported that 4 patients were withdrawn from the study because of side effects, including sustained hematologic disorders, cryptococcal infection, and cellulitis. In our study, grade 2 to 3 lymphopenia occurred, but no particular opportunistic disease prophylaxis was required; a short period of discontinuation, together with low-dose prednisone in some instances, permitted the rapid normalization of hematologic values. Moreover, there were 3 patients in which the standard 5/28-day cycle caused a grade 4 leucopenia that required a sustained treatment discontinuation. At progression in these patients, the administration of the low-dose metronomic regimen was not associated with further hematologic disorders, suggesting improved treatment tolerability compared with the standard schedule.

The last study is very recent and is that by Omuro and collegues [49]. In Phase II study, 37 glioblastoma 


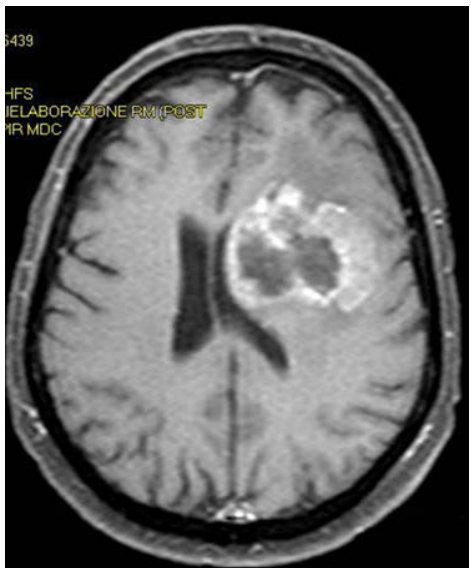

(a)

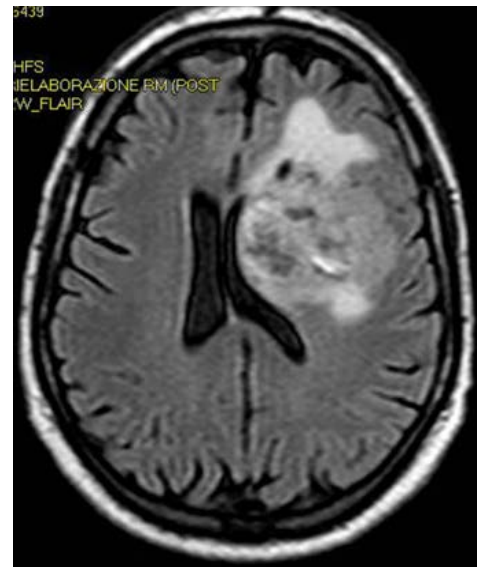

(d)

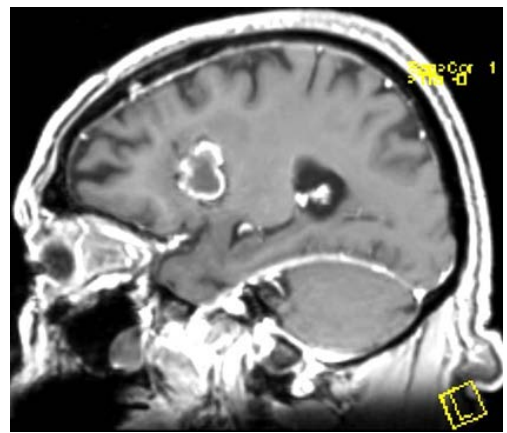

(g)

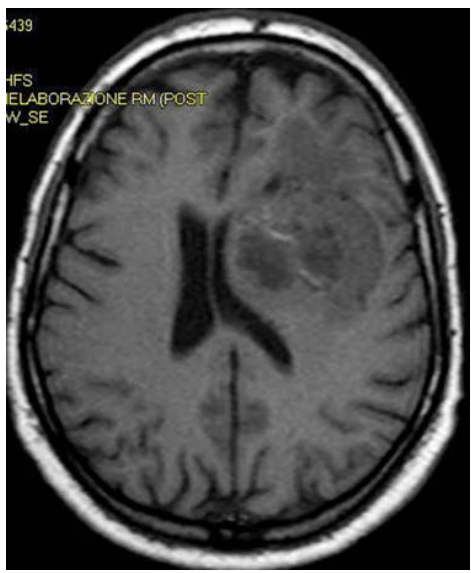

(b)

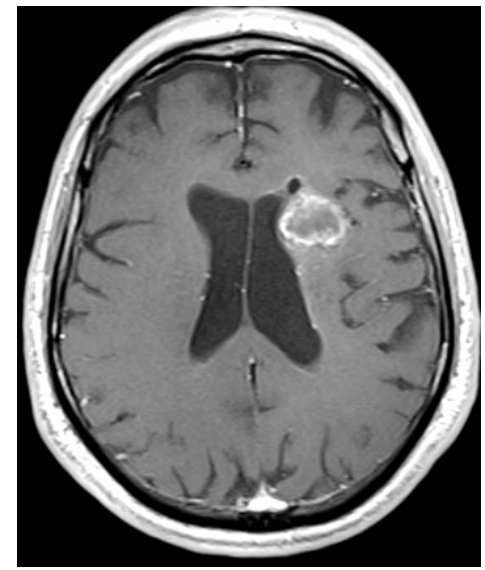

(e)

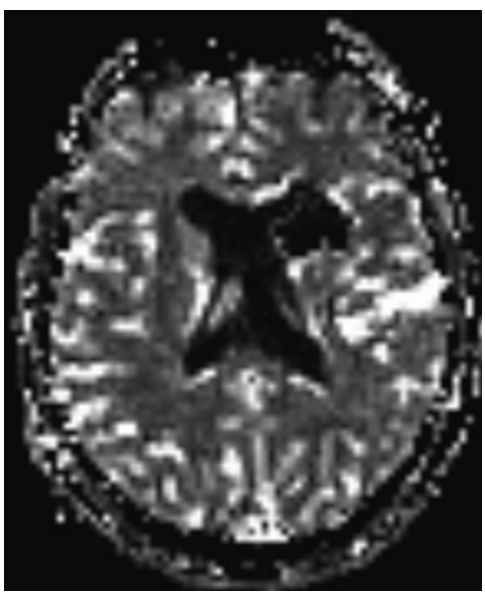

(h)

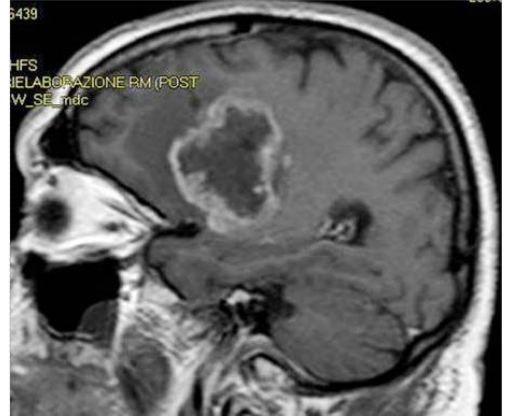

(c)

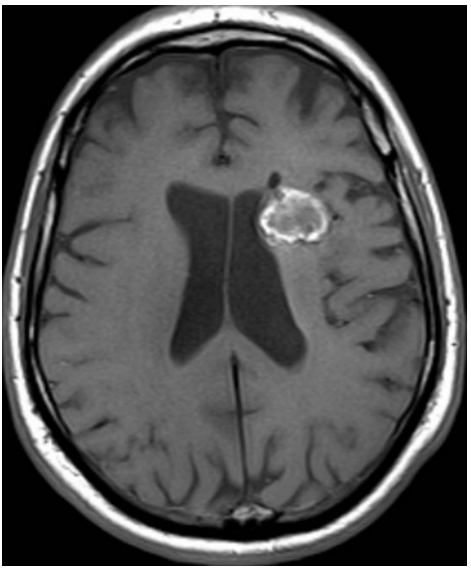

(f)

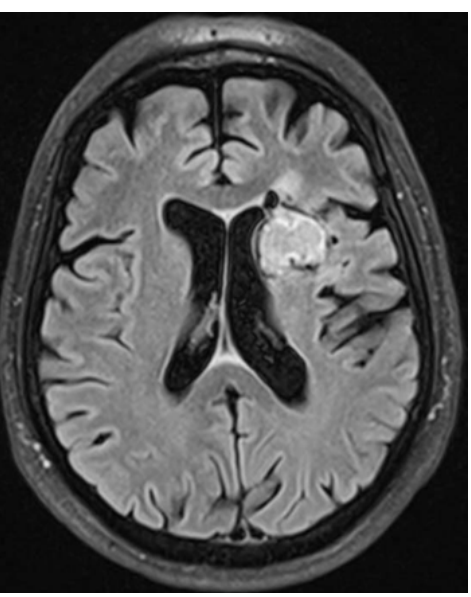

(i)

Figure 5. 60-year-old male with a left frontal glioblastoma who experienced a complete response (CR). (a)-(d): contrast enhanced MRI study obtained 40 days after completion of adjuvant concomitant radio-chemotherapy (an hyperfractionated regimen was delivered: see table 1). T1 weighted axial post-contrast (a), axial before-contrast (b), sagittal post-contrast (c) scans, together with axial T2 FLAIR scan (d), show the large necrotic tumor mass. (e)-(i): contrast-enhanced MRI study obtained after completion of 12 metronomic TMZ cycles (28 over 28 days schedule). T1 weighted axial post-contrast (e) and before contrast injection (f), together with a sagittal post-contrast image, show the presence of an hyperintense ferromagnetic ring without any enhancement. Figure $\mathrm{H}$ shows an $\mathrm{rCBV}$ map and figure I represents T2 FLAIR scan. The vast edema seems to have disappeared. Even metabolite pattern have normalized at MR spectroscopy (not shown). The patient rapidly resolved a severe right hemiparetic syndrome, with general physical improvement and a KPS that passed from 50100. 
patients were treated by metronomic daily temozolomide at the same dosage employed before. $62 \%$ of these patients have already been treated for recurrent disease, and the metronomic temozolomide represented the third line therapy. $49 \%$ of patients had received bevacizumab at first progression. These authors reported that the treatment was well tolerated, with a clinical response in $36 \%$ of patients, a PFS6 of $19 \%$ and a median OS of 7 months. These results appear noticeable, if we consider that the majority of patients was under progressive chemotherapy refractory disease. On conclusion, these authors quoted that this regimen deserves of course further investigation.

Giving the importance of MGMT status on prognosis, MGMT analysis was reported in the present series. Our data show that MGMT promoter status correlates with time to progression (Figure 3), but no correlation was found with the OS after tumor recurrence (Figure 4). The last result may be related to the fact that it has been observed that methylation status may change at recurrence [52], so it may be more fruitful to analyze MGMT expression and its impact on treatment efficacy for patients who undergo a second surgery. Moreover, we must consider that in the other cited studies [31,32], MGMT expression was not associated with differences in response to treatment, and this is consistent with the fact that the metronomic schedule was developed to overcome MGMT-related chemotherapy resistance. To confirm or reject this observation, further studies are expected.

On conclusion, the metronomic daily administration of TMZ seems to be a safe and effective option for recurrent GBM patients, especially in early recurrence. This treatment showed radiologic responses that are similar to those observed in bevacizumab-based trials, and may overcome MGMT-mediated chemotherapy resistance. Finally, the rapid effectiveness of low-dose daily TMZ administration, together with the excellent tolerability profile, make this metronomic schedule ideal for further clinical studies in combination with other chemotherapeutic agents and in the different stages in the natural history of the disease. It is noteworthy that this regimen probably received less attention than it deserves, and we hope that its wide employment in the recurrent setting will add to glioblastoma patient care.

\section{Conflict of Interest Statement}

The authors have no relevant affiliations or financial involvement with any organization or entity with a financial interest in or financial conflict with the subject matter or materials discussed in the manuscript.

\section{Funding Statement}

The authors have no funds or grants from any.

\section{REFERENCES}

[1] D. N. Louis, H. Ohgaki, O. D. Wiestler and W. K. Cavenee, "World Health Organization Classification of Tumours of the Central Nervous System," IARC Press, Lyon, 2007.

[2] R. Stupp, M. E. Hegi, M. J. van den Bent, et al., "Changing Paradigms: An Update on the Multidisciplinary Management of Malignant Glioma," Oncologist, Vol. 11, No. 2, 2006, pp. 165-180.

http://dx.doi.org/10.1634/theoncologist.11-2-165

[3] M. Salvati, A. D’Elia, A. I. Formichella and A. Frati, "Insights into Pharmacotherapy of Malignant Glioma in Adults,” Expert Opin Pharmacother Oct, Vol. 10, No. 14, 2009, pp. 2279-2290.

[4] W. Wick, M. Platten and M. Weller, "New (Alternative) Temozolomide Regimens for the Treatment of Glioma," Neuro-Oncology, Vol. 11, No. 1, 2009, pp. 69 -79. http://dx.doi.org/10.1215/15228517-2008-078

[5] M. F. Stevens, J. A. Hickman, S. P. Langdon, et al., “Antitumor Activity and Pharmacokinelics in Mice of 8-Carbamoyl-3-Methyl-Imidazo[5.I-d]-1.2.3.5-Lelrazin-4(3H)One (CCRG 81045; M \& B 39831). A Novel Drug with Potential as an Alternative to Dacarbazine," Cancer Reseach, Vol. 47, 1987, pp. 5846-5852.

[6] C. Brock, E. S. Newlands, S. R. Wedge, et al., "Phase I Trial of Temozolomide Using an Extended Continuous Oral Schedule,” Cancer Research, Vol. 58, 1998, pp. 43634367.

[7] E. S. Newlands, M. F. G. Stevens, S. R. Wedge, et al., "Temozolomide: A Review of Its Discovery, Chemical Properties, Pre-Clinical Development and Clinical Trials," Cancer Treatment Reviews, Vol. 23, No. 1, 1997, pp. 3561. http://dx.doi.org/10.1016/S0305-7372(97)90019-0

[8] R. Stupp, W. Mason, M. van den Bent, et al., "Rariotherapy plus Concomitant and Adjuvant Temozolomide for Glioblastoma," The New England Journal of Medicine, Vol. 352, No. 10, 2005, pp. 987-996.

http://dx.doi.org/10.1056/NEJMoa043330

[9] W. Wick, J. P. Steinbach, W. M. Kuker, et al., "One Week on/One Week off: A Novel Active Regimen for Temozolomide for Recurrent Glioblastoma,” Neurology, Vol. 62, No. 11, 2004, pp. 2113-2115.

http://dx.doi.org/10.1212/01.WNL.0000127617.89363.84

[10] A. A. Brandes, A. Tosoni, G. Cavallo, et al., "Temozolomide 3 Weeks on and 1 Week off as First-Line Therapy for Recurrent Glioblastoma: Phase II Study from Gruppo Italiano Cooperative Dineuro-Oncologia (GICNO),” British Journal of Cancer, Vol. 95, 2006, pp. 1155-1160. http://dx.doi.org/10.1038/sj.bjc.6603376

[11] A. Tosoni, G. Cavallo, M. Ermani, et al., "Is Protracted Low-Dose Temozolomide Feasible in Glioma Patients," Neurology, Vol. 66, No. 3, 2006, pp. 427-429. http://dx.doi.org/10.1212/01.wnl.0000196465.83423.ec

[12] E. Franceschi, A. Omuro, A. Lassman, et al., "Salvage Temozolomide for Prior Temozolomide Responders," Cancer, Vol. 104, No. 11, 2005, pp. 2473-2476. http://dx.doi.org/10.1002/cncr.21564 
[13] J. Perry, P. Rizek, R. Cashman, M. Morrison and T. Morrison, "Temozolomide Rechallenge in Recurrent Malignant Glioma by Using a Continuous Temozolomide Schedule: The 'Rescue' Approach,” Cancer, Vol. 113, No. 8, 2008, pp. 2152-2157. http://dx.doi.org/10.1002/cncr.23813

[14] M. Weller, “Temozolomide and MGMT Forever?” Neuro-Oncology, Vol. 12, No. 3, 2010, pp. 219-220. http://dx.doi.org/10.1093/neuonc/noq016

[15] M. E. Hegi, L. Liu, J. Herman, et al,. "Correlation of O6-Methylguanine Methyltransferase (MGMT) Promoter Methylation with Clinical Outcomes in Glioblastoma and Clinical Strategies to Modulate MGMT Activity,” Journal of Clinical Oncology, Vol. 26, No. 25, 2008, pp. 4189-4199. http://dx.doi.org/10.1200/JCO.2007.11.5964

[16] M. Esteller, J. Garcia-Foncillas, E. Andion, et al., "Inactivation of the DNA-Repair Gene MGMT and the Clinical Response of Gliomas to Alkylating Agents," The New England Journal of Medicine, Vol. 343, No. 19, 2000, pp. 1350-1354. http://dx.doi.org/10.1056/NEJM200011093431901

[17] M. E. Hegi, A.-C. Diserens, S. Godard, et al., "Clinical Trial Substantiates the Predictive Value of O-6-Methylguanine-DNA Methyltransferase Promoter Methylation in glioblastoma Patients Treated with Temozolomide," Clinical Cancer Research, Vol. 10, 2004, pp. 1871-1874. http://dx.doi.org/10.1158/1078-0432.CCR-03-0384

[18] C. Schold, D. Kokkinakis, S. Chang, et al., " $\mathrm{O}^{6}$-Benzylguanine Suppression of $\mathrm{O}^{6}$-Alkylguanine-DNA Alkyltransferase in Anaplastic Gliomas,” Neuro-Oncology, Vol. 6, No. 1, 2004, pp. 28-32. http://dx.doi.org/10.1215/S115285170300019X

[19] L. Karayan-Tapon, V. Quillien, J. Guilhot, et al., "Prognostic Value of $\mathrm{O}^{6}$-Methylguanine-DNA Methyltransferase Status in Glioblastoma Patients, Assessed by Five Different Methods,” Journal of Neuro-Oncology, Vol. 97, No. 3, 2010, pp. 311-322. http://dx.doi.org/10.1007/s11060-009-0031-1

[20] A. W. Tolcher, S. L. Gerson, L. Denis, et al., "Marked inactivation of $\mathrm{O}^{6}$-Alkylguanine-DNA Alkyltransferase Activity with Protracted Temozolomide Schedules," British Journal of Cancer, Vol. 88, No. 7, 2003, pp. 10041011. http://dx.doi.org/10.1038/sj.bjc.6600827

[21] K. H. Plate and W. Risau, "Angiogenesis in Malignant Gliomas,” Glia, Vol. 15, No. 3, 1995, pp. 339-347. http://dx.doi.org/10.1002/glia.440150313

[22] S. P. Leon, R. D. Folkerth and P. M. Black, "Microvessel Density Is a Prognostic Indicator for Patients with Astroglial Brain Tumors,” Cancer, Vol. 77, No. 2, 1996, pp. 362-372.

http://dx.doi.org/10.1002/(SICI)1097-0142(19960115)77: 2<362::AID-CNCR20>3.0.CO;2-Z

[23] J. Dietrich, A. Norden and P. Wen, "Emerging Antiangiogenic Treatments for Gliomas-Efficacy and Safety Issues. Review," Current Opinion in Neurology, Vol. 21, No. 6, 2008, pp. 736-744. http://dx.doi.org/10.1097/WCO.0b013e3283131370

[24] S. Kesari, D. Schiff, L. Doherty, et al., "Phase II Study of
Metronomic Chemotherapy for Recurrent Malignant Gliomas in Adults," Neuro-Oncology, Vol. 9, No. 3, 2007, pp. 354-363.

http://dx.doi.org/10.1215/15228517-2007-006

[25] R. S. Kerbel and B. A. Kamen, "The Anti-Angiogenic Basis of Metronomic Chemotherapy,” Nature Reviews Cancer, Vol. 4, 2004, pp. 423-436. http://dx.doi.org/10.1038/nrc1369

[26] M. W. Kieran, C. D. Turner, J. B. Rubin, et al., “A Feasibility Trial of Antiangiogenic (Metronomic) Chemotherapy in Pediatric Patients with Recurrent or Progressive Cancer," Journal of Pediatric Hematology/Oncology, Vol. 27, No. 11, 2005, pp. 573-581. http://dx.doi.org/10.1097/01.mph.0000183863.10792.d4

[27] J. Tuettenberg, R. Grobholz, T. Korn, F. Wenz, R. Erber and P. Vajkoczy, "Continuous Low-Dose Chemotherapy plus Inhibition of Cyclooxygenase-2 as an Antiangiogenic Therapy of Glioblastoma Multiforme,” Journal of Cancer Research and Clinical Oncology, Vol. 131, No. 1, 2005, pp. 31-40.

http://dx.doi.org/10.1007/s00432-004-0620-5

[28] F. Bertolini, S. Paul, P. Mancuso, et al., "Maximum Tolerable Dose and Low-Dose Metronomic Chemotherapy Have Opposite Effects on the Mobilization and Viability of Circulating Endothelial Progenitor Cells," Cancer Reseach, Vol. 63, 2003, pp. 4342-4346.

[29] G. Bocci, G. Francia, S. Man, et al., "Thrombospondin-1, a Mediator of the Antiangiogenic Effects of Low-Dose Metronomic Chemotherapy," Proceedings of the National Academy of Sciences of the United States of America, Vol. 100, No. 22, 2003, pp. 12917-12922. http://dx.doi.org/10.1073/pnas.2135406100

[30] J. Perry, K. Belanger, W. Mason, et al., "Phase II Trial of Continuous Dose-Intense Temozolomide in Recurrent Malignant Glioma: RESCUE Study,” Journal of Clinical Oncology, Vol. 28, No. 12, 2010, pp. 2051-2057.

http://dx.doi.org/10.1200/JCO.2009.26.5520

[31] D. S. Kong, J. I. Lee, J. H. Kim, S. T. Kim, W. S. Kim, Y. L. Suh, S. M. Dong and D. H. Nam, "Phase II Trial of Low-Dose Continuous (Metronomic) Treatment of Temozolomide for Recurrent Glioblastoma,” Neuro-Oncology, Vol. 12, No. 3, 2010, pp. 289-296. http://dx.doi.org/10.1093/neuonc/nop030

[32] W. B. Pope, A. Lai, P. Nghiemphu, P. Mischel and T. F. Cloughesy, "MRI in Patients with High-Grade Gliomas Treated with Bevacizumab and Chemotherapy,” Neurology, Vol. 66, No. 8, 2006, pp. 1258-1260. http://dx.doi.org/10.1212/01.wnl.0000208958.29600.87

[33] A. D. Norden, G. S. Young, K. Setayesh, A. Muzikansky, R. Klufas, G. L. Ross, A. S. Ciampa, L. G. Ebbeling, B. Levy, J. Drappatz, S. Kesari and P. Y. Wen, "Bevacizumab for Recurrent Malignant Gliomas: Efficacy, Toxicity, and Patterns of Recurrence," Neurology, Vol. 70, No. 10, 2008, pp. 779-787. http://dx.doi.org/10.1212/01.wnl.0000304121.57857.38

[34] J. Vredenburgh, A. Desjardins, J. E. Herndon II, J. M. Dowell, D. A. Reardon, J. A. Quinn, J. N. Rich, S. Sathornsumetee, S. Gururangan, M. Wagner, D. D. Bigner, A. H. 
Friedman and H. S. Friedman, "Phase II Trial of Bevacizumab and Irinotecan in Recurrent Malignant Glioma,” Clinical Cancer Research, Vol. 13, No. 30, 2007, pp. 1253-1259.

http://dx.doi.org/10.1158/1078-0432.CCR-06-2309

[35] T. Kreisl, L. Kim, K. Moore, P. Duic, C. Royce, I. Stroud, N. Garren, M. Mackey, J. A. Butman, K. Camphausen, J. Park, P. S. Albert and H. A. Fine, "Phase II Trial of SingleAgent Bevacizumab Followed by Bevacizumab Plus Irinotecan at Tumor Progression in Recurrent Glioblastoma," Journal of Clinical Oncology, Vol. 27, No. 5, 2009, pp. 740-745. http://dx.doi.org/10.1200/JCO.2008.16.3055

[36] D. R. Macdonald, T. L. Cascino, S. C. Schold Jr. and J. G. Cairncross, "Response Criteria for Phase II Studies of Supratentorial Malignant Glioma," Journal of Clinical Oncology, Vol. 8, No. 7, 1990, pp. 1277-1280.

[37] J. Tuettenberg, R. Grobholz, M. Seiz, M. A. Brockmann, F. Lohr, F. Wenz and P. Vajkoczy, "Recurrence Pattern in Glioblastoma Multiforme Patients Treated with Anti-Angiogenic Chemotherapy," Journal of Cancer Research and Clinical Oncology, Vol. 135, No. 9, 2009, pp. 12391244. http://dx.doi.org/10.1007/s00432-009-0565-9

[38] E. S. Newlands., G. R. Blackledge, J. A. Slack, G. J. Rustin, D. B. Smith, N. S. Stuart, C. P. Quarterman, R. Hoffman, M. F. G. Stevens and M. H. Brampton, "Phase I Trial of Temozolomide (CCRG 81045: M\&B 39831: NSC 362856),” British Journal of Cancer, Vol. 65, No. 2, 1992, pp. 287-291. http://dx.doi.org/10.1038/bjc.1992.57

[39] P. Y. Wen, A. D. Norden, J. Drappatz and E. Quant, "Response Assessment Challenges in Clinical Trials of Gliomas,” Current Oncology Reports, Vol. 12, No. 1, 2010, pp. 68-75. http://dx.doi.org/10.1007/s11912-009-0078-3

[40] E. T. Wong, K. R. Hess, M. J. Gleason, K. A. Jaeckle, A. P. Kyritsis, M. D. Prados, V. A. Levin and W. K. Yung, "Outcomes and Prognostic Factors in Recurrent Glioma Patients Enrolled onto Phase II Clinical Trials,” Journal of Clinical Oncology, Vol. 17, No. 8, 1999, pp. 25722578.

[41] K. Lamborn, A. Yung, S. Chang, P. Y. Wen, T. F. Cloughesy, L. M. DeAngelis, H. I. Robins, F. S. Lieberman, H. A. Fine, K. L. Fink, L. Junck, L. Abrey, M. R. Gilbert, M. Mehta, J. G. Kuhn, K. D. Aldape, J. Hibberts, P. M. Peterson, M. D. Prados and North American Brain Tumor Consortium, "Progression-Free Survival: An Important End Point in Evaluating Therapy for Recurrent High-Grade Gliomas,” Neuro-Oncology, Vol. 10, No. 2, 2008, pp. 162-170. http://dx.doi.org/10.1215/15228517-2007-062

[42] M. C. Chamberlain, M. J. Glantz, L. Chalmers, A. Van Horn and A. E. Sloan, "Early Necrosis Following Temodar and Radiotherapy in Patients with Glioblastoma," Journal of Neuro-Oncology, Vol. 82, No. 1, 2007, pp. 8183. http://dx.doi.org/10.1007/s11060-006-9241-y

[43] D. Brandsma, L. Stalpers, W. Taal, P. Sminia and M. J. van den Bent, "Clinical Features, Mechanisms, and Management of Pseudoprogression in Malignant Gliomas,”
Lancet Oncology, Vol. 9, No. 5, 2008, pp. 453-461. http://dx.doi.org/10.1016/S1470-2045(08)70125-6

[44] W. Taal, D. Brandsma, H. G. de Bruin, J. E. Bromberg, A. T. Swaak-Kragten, P. A. E. S. Smitt, C. A. van Es and M. J. van den Bent, "Incidence of Early Pseudo-Progression in a Cohort of Malignant Glioma Patients Treated with Chemoirradiation with Temozolomide," Cancer, Vol. 113, No. 2, 2008, pp. 405-410.

http://dx.doi.org/10.1002/cncr.23562

[45] A. A. Brandes, A. Tosoni, F. Spagnolli, et al., "Disease Progression or Pseudoprogression after Concomitant Radiochemotherapy Treatment: Pitfalls in Neurooncology," Neuro-Oncology, Vol. 10, No. 3, 2008, pp. 361-367. http://dx.doi.org/10.1215/15228517-2008-008

[46] P. Y. Wen, D. R. Macdonald, D. A. Reardon, et al., "Proposal for an Updated Response Criteria in High-Grade Gliomas,” Journal of Clinical Oncology (in press).

[47] R. F. Barajas Jr., J. S. Chang, M. R. Segal, A. T. Parsa, M. W. McDermott, M. S. Berger and S. Cha, "Differentiation of Recurrent Glioblastoma Multiforme from Radiation Necrosis after External Beam Radiation Therapy with Dynamic Susceptibility Weighted Contrast-Enhanced Perfusion MR Imaging,” Radiology, Vol. 253, No. 2, 2009, pp. 486-496. http://dx.doi.org/10.1148/radiol.2532090007

[48] L. S. Hu, L. C. Baxter, K. A. Smith, B. G. Feuerstein, J. P. Karis, J. M. Eschbacher, S. W. Coons, P. Nakaji, R. F. Yeh, J. Debbins and J. E. Heiserman, "Relative Cerebral Blood Volume Values to Differentiate High-Grade Glioma Recurrence from Posttreatment Radiation Effect: Direct Correlation between Image-Guided Tissue Histopathology and Localized Dynamic Susceptibility-Weighted Contrast-Enhanced Perfusion MR Imaging Measurements,” American Journal of Neuroradiology, Vol. 30, No. 3, 2009, pp. 552-558. http://dx.doi.org/10.3174/ajnr.A1377

[49] E. Matsusue, J. R. Fink, J. K. Rockhill, T. Ogawa and K. R. Maravilla, "Distinction between Glioma Progression and Post-Radiation Change by Combined Physiologic MR Imaging," Neuroradiology, Vol. 52, No. 4, 2010, pp. 297306. http://dx.doi.org/10.1007/s00234-009-0613-9

[50] A. A. Brandes, E. Franceschi, A. Tosoni, S. Bartolini, A. Bacci, R. Agati, C. Ghimenton, S. Turazzi, A. Talacchi, M. Skrap, G. Marucci, L. Volpin, L. Morandi, S. Pizzolitto, M. Gardiman, A. Andreoli, F. Calbucci and M. Ermani, " $\mathrm{O}^{6}$-Methylguanine DNA-Methyltransferase Methylation Status Can Change between First Surgery for Newly Diagnosed Glioblastoma and Second Surgery for Recurrence: Clinical Implications,” Neuro-Oncology, Vol. 12, No. 3, 2010, pp. 283-288.

http://dx.doi.org/10.1093/neuonc/nop050

[51] A. Omuro, T. Chan, L. Abrey, M. Khasraw, A. S. Reiner, T. J. Kaley, L. M. DeAngelis, A. B. Lassman, C. P. Nolan, I. T. Gavrilovic, A. Hormigo, C. Salvant, A. Heguy, A. Kaufman, J. T. Huse, K. S. Panageas, A. F. Hottinger and I. Mel, "Phase II Trial of Continuous Low Dose Temozolomide for Patients with Recurrent Malignant Glioma," Neuro-Oncology, Vol. 15, No. 2, 2013, pp. 242-250. http://dx.doi.org/10.1093/neuonc/nos295 